\title{
Virtual Biology: Teaching Histology in the Age of Facebook
}

\author{
Michael Hortsch ${ }^{1}$ \\ University of Michigan, Department of Cell and Developmental Biology, Ann Arbor, Michigan, USA
}

Ever since ANTon van Leeuwenhoek (1632-1723) used his simple magnifying devices for viewing biological material, the study of cells and tissues at the microscopic scale has largely been driven by technological advances. In the mid-19th century, the collaboration among Carl Zeiss (1816-1888), Ernst Abbe (18401905), and Otto Schott (1851-1935) made the industrial manufacturing of high-quality microscopes possible, placing them into the hands of a larger number of scientists and triggering an avalanche of new discoveries that laid the foundation for the fields of histology/ microanatomy and later, cell biology. During the 20th century, new technologies, such as the invention of the electron microscope by Ernst Ruska (1906-1988) and epifluorescence microscopy, advanced our knowledge about biological structures to even smaller dimensions. Over the last 30 years, a flurry of new microscopic techniques, such as the use of viable fluorescent dyes, like green fluorescence protein, and the invention of confocal, two-photon excitation, atomic force, and fluorescence resonance energy transfer microscopy, just to name a few, further accelerated this development. This technological revolution in the biomedical experimental sciences is now mirrored by a similar revolution in how we teach microscopic biology to our students.

\section{Virtual Microscopy}

For many years, the teaching of histology or microanatomy has relied on banks of light microscopes and boxes of histological glass slides. This gave students an opportunity to learn how to operate a light microscope. However, this approach suffered from a great variability in the quality of the slide material. In addition, many tissues and organ preparations are difficult to come by, and glass slides are inherently breakable. These problems and the upkeep of many light microscopes are a continuous expense, especially during times of diminishing financial support for higher education. Over the last 10 years, many schools and universities in the United States and in other countries have moved away from the use of real microscopes and have adapted a novel, electronic way to expose students to the structure of cells and tissues at the microscopic scale $(1,2)$. "Virtual microscopy" uses computer screens that are linked to servers hosting high-resolution digitized scans of histological slides. Similar to the Google Earth concept, students can move to different areas of a slide and zoom in or out at their own choosing (Fig. 1). As the best slide preparations can be selected for digitization, all students have access to the same high-quality material. In addition, the digital image files can easily be duplicated and exchanged among owners of virtual slide collections. The use of virtual slides also frees students from restricted classroom and laboratory hours, as they are able to access the material on their own time and from any location with a sufficiently fast internet connection. This ability is highly appreciated by today's generation of students. On the negative side, students will not experience the variability of biological material and preparations. Also, virtual microscopy does not allow them to gain a glimpse of the threedimensionality of tissues that can be achieved by adjusting the fine-focus knob of a real microscope. By definition, the virtual microscopy approach is highly dependent on a fast and stable technological infrastructure. Instead of large numbers of microscopes and slide boxes, schools or students have to provide computers, and servers with appropriate software are required for the hosting of large quantities of data. Even the use of redundant servers does not guarantee continuous service, as computers and servers need constant software upgrades and are prone to crashes.

\section{Changes in Teaching Microscopic Biology}

Our experiences at the University of Michigan provide an illuminating example how the use of traditional teaching methods in combination with electronic resources, such as virtual microscopy, has changed the teaching of microscopy biology. Virtual microscopy together with traditional microscopes and slide boxes were initially used in the first year of the Michigan medical curriculum during the academic year of 2005/ 06. Starting the following academic year, all medical and dental, as well as graduate and undergraduate, histology teaching at the University of Michigan relied almost entirely on virtual images. Although all Michigan medical students were offered loan microscopes

\footnotetext{
1 Correspondence: University of Michigan, Dept. of Cell and Developmental Biology, 109 Zina Pitcher Pl., Ann Arbor, MI 48109-2200, USA. E-mail: hortsch@umich.edu

doi: 10.1096/fj.13-0201ufm
} 


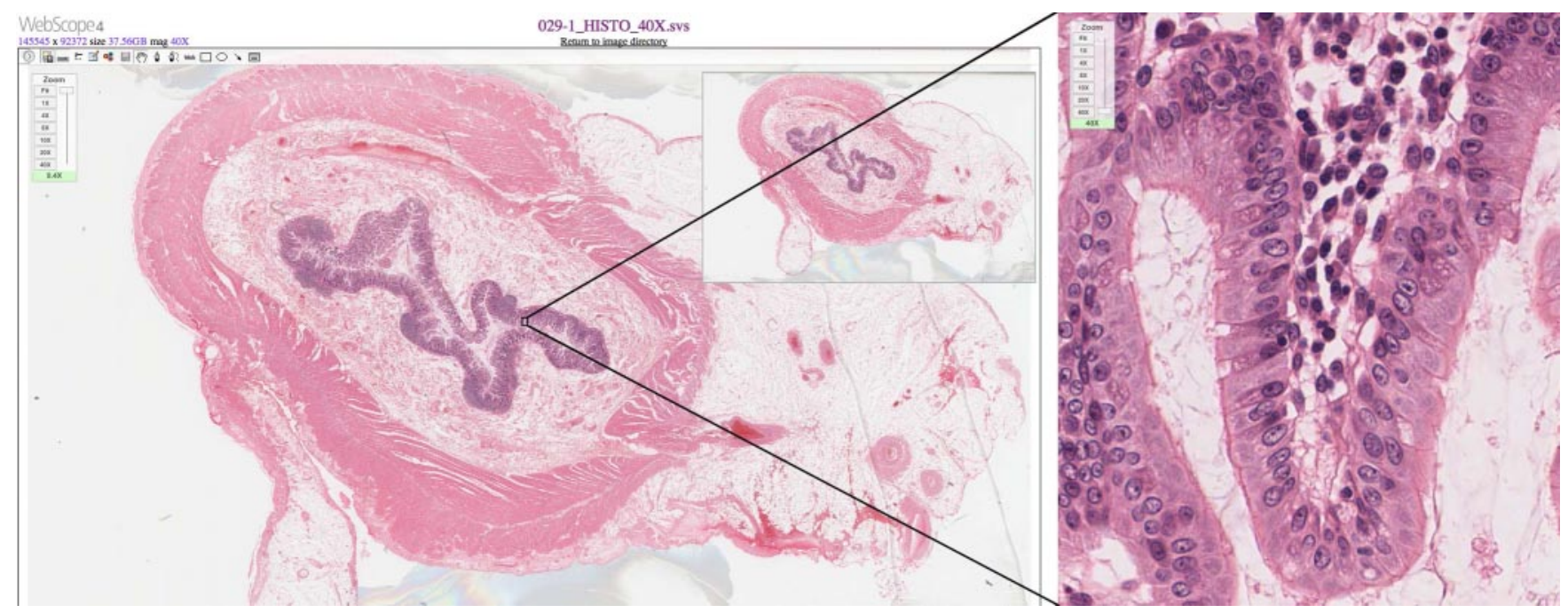

Figure 1. Virtual microscope screen capture of a cross section of a jejunum monkey (http://141.214.65.171/Histology/ Basic\%20Tissues/Epithelium\%20and\%20CT/029-1_HISTO_40X.svs/view.apml). The left side represents a virtual slide from the Michigan Histology Virtual Slide collection at $0.4 \times$ magnification. The right panel displays a subarea of the section shown on the left at maximal magnification $(40 \times)$.

and histological slide sets during the last 6 years, of over 1000 first-year medical students, $<10$ made use of this option. Over the last 2 years, we offered the use of seven to eight demonstration microscopes with selected histological glass slides in one of the histology laboratories. More than $65 \%$ of all students reported that they never took advantage of this opportunity. Clearly, most students are not motivated to learn traditional microscope techniques and exclusively use the electronic option that is provided by the virtual slide collection. The reason for this preference appears to be multifold: primarily, a matter of convenience and of the timedemanding schedule that is associated with today's college and graduate curricula.

Access to the Michigan virtual slides is provided by three course websites (3) that also contain learning objectives, laboratory assignments, virtual electronic micrographs, and sample test questions, as well as a plethora of supplemental resources, such as lecture handouts, slide orientation, and review PowerPoint files. A majority of Michigan students still prefer to attend histological lectures in person $(\sim 43 \%$ of medical students report that they always attend the histology lectures), rather than watching downloadable podcasts of the lectures. Overall, all electronic resources, including virtual slides, enjoy an increased use over the course of the academic year, whereas the use of scheduled didactic opportunities, such as lectures and laboratories, as well as other traditional learning resources, especially textbooks, is declining over time (unpublished results).

The still relatively new approach of studying microscopic tissue structures by virtual microscopy also has the potential to be integrated into the open-source platforms that are becoming available for delivering higher educational course material online. As the Michigan virtual slide collection is published under a Creative Commons BY-NC-SA license, it is freely accessible worldwide via one of the Michigan histology websites. In addition, virtual microscope slides can easily be adapted and integrated into online college and graduate-level educational collections, which are currently being developed, including Connexions, Coursera, edX, iTunes U, and others (4).

However, are these new ways of disseminating scientific knowledge and developing analytic skills more effective and beneficial to our students? A lecture does not become better just because it is streamed as a podcast via the internet. A great lecture is more than just a series of projected lecture slides and the voice of the presenter in the background. Actually, one can reason that such delivery modus must be lacking, as it is often devoid of nonverbal lines of communication that a good lecturer will use to capture his/her audience. Similarly, we need to question whether the delivery of histology by virtual microscopy is an effective method for our students to learn tissue and organ organization and histological concepts. Publications on the effectiveness of virtual microscopy as a teaching tool vary in their conclusions, with some reporting none and many showing significant improvements in students' performance (5-7).

Considering the increasing use of networked digital medical data-keeping and -exchange in the clinical arena, one might argue that a student in a biomedical field does not need the knowledge of how to operate a light microscope to become a competent health-care provider. However, especially in rural areas and developing countries or in special clinical environments, e.g., an OB/GYN practice, the use of a simple light microscope is still an important part of patient care (8). Therefore, it is fair to contend that the ability of using a light microscope is still a useful, although not an essential, skill for a practicing physician. Medical students, who need to use microscopes in the clinics, will probably become proficient later during their clinical 
education. Equally, those students, who will be entering into a career of experimental research, will acquire the necessary microscope skills quickly in a laboratory environment.

\section{Additional, New Teaching Technologies}

From our experiences with using virtual microscopy for the learning of histology, it is clear that the current Facebook generation of students is willing and eager to embrace new technologies, especially if they are interactive. As more and more course material is issued in the form of PowerPoint and PDF files, many educational institutions require students to have laptop computers. In addition, more examinations are now administrated using electronic testing systems, and the next generation of electronic devices is already invading our classrooms in the form of iPads and Android-based tablets. With built-in wireless connectivity, these gadgets make all kinds of information and educational tools extremely portable and ubiquitously accessible. Unfortunately, the development and supply of high-quality educational resources for these devices are lagging behind. Good eBooks with additional electronic features are still scarce, and among the flood of iPad and Android applications, too few serve the higher education and research community. The ability to view virtual histology slides with tablet readers is still cumbersome, but with new software becoming available, this rapidly becomes a problem of the past. In addition, these instruments offer many possibilities of developing new, interactive educational resources that have not been available before and serve specific didactic needs. As histology is a very visual subject, tablet readers are ideal tools for studying histological images. We recently published an iPad application called "Michigan eHistology-A SecondLook Series" (9) that can be used as a self-evaluation tool by students, who learn histology and want to test their level of preparedness before taking quizzes and examinations.

An additional aspect of these technological advances in teaching microscopic biology is their worldwide availability, which allows instructional institutions in developing countries to take an educational leapfrog by providing modern, highly efficient didactic resources to their students via the internet. Again, this is exemplified by the use of the Michigan histology course websites. In response to the three histology course websites, we are regularly receiving requests from teaching faculty all over the world, who are interested in using or implementing virtual microscopy for their histology courses. Of the 33,180 visits that the three Michigan Histology course websites received during the month of October 2012, $45.7 \%$ came from outside of the United States, preferentially from English-speaking countries, such as the United Kingdom, Canada, and Australia. However, the list also contains a significant number of non-English-speaking developing countries, such as the Philippines, India, Malaysia, Colombia, Brazil, Egypt, and many more. This indicates that despite some language barriers, this kind of open resource material is frequently used at locations that often might not have adequate resources for teaching students microanatomy with light microscopes and expensive glass slide sets.

The development of new teaching technologies and their implementation in the biological sciences are inevitable and are already progressing at a rapid speed. It will remain our responsibility as educators to efficiently coordinate them with traditional teaching methods and to integrate them into existing curricula for the benefit of our students. Our students are willing and prepared to embrace these new technologies. Are we, as their teachers, equally ready?

\section{REFERENCES}

1. Drake, R. L., McBride, J. M., Lachman, N., and Pawlina, W. (2009) Medical education in the anatomical sciences: the winds of change continue to blow. Anat. Sci. Educ. 2, 253-259

2. Coleman, R. (2009) Can histology and pathology be taught without microscopes? The advantages and disadvantages of virtual histology. Acta Histochem. 111, 1-4

3. University of Michigan Medical School. Histology and Microscopy Learning Resources, http://histology.med.umich.edu/schedule/ medical, /dental or /undergraduate-graduate

4. Rice University. Connexions, http://cnx.org/; Coursera, https:// www.coursera.org/; edX, http://www.edxonline.org/; iTunes U. Apple in Education, http://www.apple.com/education/itunes-u/

5. Husmann, P. R., O'Loughlin, V. D., and Braun, M. W. (2009) Quantitative and qualitative changes in teaching histology by means of virtual microscopy in an introductory course in human anatomy. Anat. Sci. Educ. 2, 218-226

6. Triola, M. M., and Holloway, W. J. (2011) Enhanced virtual microscopy for collaborative education. BMC Med. Educ. 11, 4

7. Kumar, R. K., Freeman, B., Velan, G. M., and De Permentier, P. J. (2006) Integrating histology and histopathology teaching in practical classes using virtual slides. Anat. Rec. B New Anat. 289, $128-133$

8. Pratt, R. L. (2009) Are we throwing histology out with the microscope? A look at histology from the physician's perspective. Anat. Sci. Educ. 2, 205-209

9. University of Michigan Medical School. Michigan eHistology-A SecondLook Series, http://www.med.umich.edu/lrc/second look/

The opinions expressed in editorials, essays, letters to the editor, and other articles comprising the Up Front section are those of the authors and do not necessarily reflect the opinions of FASEB or its constituent societies. The FASEB Journal welcomes all points of view and many voices. We look forward to hearing these in the form of op-ed pieces and/or letters from its readers addressed to journals@faseb.org. 